\title{
ÉTUDE EXPÉRIMENTALE DE LA COMPÉTITION INTERSPÉCIFIQUE ENTRE JUVÉNILES DE SAUMON COHO, ONCORHYNCHUS KISUTCH, ET DE SAUMON ATLANTIQUE, SALMO SALAR, EN EAU DOUCE.
}

\author{
M. HELAND et E. BEALL
}

INRA, Station d'Hydrobiologie, Unité Ecologie des Poissons, B.P. 3, 64310 Saint-Pée-sur-Nivelle, France.

\section{RÉSUMÉ}

Le risque d'introduction accidentelle du saumon coho dans les rivières peuplées par le saumon atlantique a conduit à développer des recherches en milieu expérimental sur les mécanismes de la compétition entre alevins et juvéniles des deux espèces. Les conséquences possibles de cette compétition ont été évaluées en termes de survie, croissance, prédation, dévalaison et occupation de l'habitat.

Les résultats montrent que la présence des saumons cohos n'a pas une influence très marquée sur les alevins ou juvéniles de saumon atlantique si l'environnement trophique est favorable. La microdistribution est différente, les saumons atlantiques occupant plutôt les radiers alors que les saumons cohos préfèrent les profonds. Le comportement pélagique et grégaire des jeunes saumons cohos, nettement plus gros que les saumons atlantiques du même âge dès l'émergence, peut être opposé au comportement benthique et inféodé au substrat de ces derniers. Ces différences s'accentuent en sympatrie, aux dépens du saumon atlantique, désavantagé par la taille. Sous conditions trophiques limitantes, la croissance et la sédentarité des alevins de saumon atlantique sont significativement affectées par la présence des cohos, ce qui justifie d'émettre d'expresses réserves sur l'intérêt de l'introduction du saumon coho dans les rivières françaises.

\section{EXPERIMENTAL STUDY OF INTERSPECIFIC COMPETITION BETWEEN COHO SALMON, ONCORHYNCHUS KISUTCH, AND ATLANTIC SALMON, SALMO SALAR, JUVENILES IN FRESHWATER.}

\begin{abstract}
The risk of accidental introduction of coho salmon in rivers inhabited by Atlantic salmon induced research on competition mechanisms between fry and juveniles of the two species under experimental conditions. Possible consequences of the competition were evaluated in terms of survival, growth, predation, downstream movement and habitat use.

Results showed that under good trophic conditions, the presence of coho salmon had no influence on Atlantic salmon fry and juveniles. Microdistribution was different, Atlantic salmon holding position in riffles and coho salmon preferring pools. The pelagic and gregarious behaviour of coho salmon juveniles, that are much larger than Atlantic salmon at the same stage after emergence, contrasted with the benthic substrate-bound behaviour of the latter. These differences increased in sympatry at the expense of Atlantic salmon, which was handicapped by its smaller size. Under limiting trophic conditions, the growth and sedentary behaviour of Atlantic salmon were significantly altered by the presence of coho salmon, thus justifying explicit doubt on the interest of introducing coho salmon into French rivers.
\end{abstract}




\section{INTRODUCTION}

Au cours des années 1970, avec l'accroissement des importations d'oeufs de saumon coho (Oncorhynchus kisutch) en Europe et notamment en France, en vue d'en développer l'aquaculture, le risque d'introduction accidentelle de cette espèce dans le milieu naturel était devenu préoccupant (SOLOMON, 1979; EUZENAT et FOURNEL, 1981). L'évaluation de ce risque et des conséquences de cette introduction vis-à-vis des populations résidentes, comme celles de saumon atlantique, Salmo salar, est apparue comme indispensable aux gestionnaires. Dans ce but, des expériences en milieu contrôlé ont été conduites à l'INRA avec, à l'époque, le soutien financier du CNEXO.

Le saumon coho a un cycle vital proche de celui du saumon atlantique ou de la truite de mer (Salmo trutta L.). II reste de 3 à 18 mois dans les parties amont des rivières et en particulier dans les zones de faciès lotique, avant de partir en migration vers la mer. L'éventuelle influence défavorable de cette espèce sur les Salmonidés migrateurs autochtones s'exercerait, le cas échéant, au niveau des relations interspécifiques pendant la phase potamique du cycle vital, c'est-à-dire au moment de la reproduction suivie du développement des juvéniles dans le même milieu trophique.

En conséquence, les recherches se sont orientées vers l'analyse des interactions comportementales entre le saumon coho et le saumon atlantique durant la phase la plus critique du cycle, qui correspond à l'émergence et la dispersion initiale. Au cours de cette phase, les alevins en interaction sociale avec leurs congénères et les autres espèces colonisent l'espace disponible et plus particulièrement le microhabitat qui correspond le mieux à leurs aptitudes (HELAND et al., 1995). Une première synthèse de quatre saisons d'expérimentations est présentée ici en s'appuyant sur des résultats inédits et en faisant référence autant que possible aux résultats partiels déjà publiés.

\section{MATÉRIEL ET MÉTHODES}

Sur un plan général, les expériences ont été réalisées dans différentes structures expérimentales complémentaires : ruisseau semi-naturel extérieur de grandes dimensions, ruisseaux artificiels de laboratoire et aquariums à courant d'eau. Les analyses étaient presque toutes basées sur le même principe : comparaison de lots de saumon atlantique ou de saumon coho seuls ou en sympatrie dans les secteurs identiques d'un même ruisseau ou dans des ruisseaux (ou aquariums) distincts mais comparables.

La plupart des expérimentations ont été réalisées dans un ruisseau artificiel extérieur, le chenal de frai du Lapitxuri, alimenté par dérivation d'un ruisseau naturel, en vue de déterminer l'influence des alevins ou juvéniles d'une espèce sur la survie, l'émigration amont ou aval, la sédentarité, le choix d'habitat, la croissance et la production d'autres juvéniles ou alevins de la même ou de l'autre espèce. Les interactions comportementales ont été observées conjointement dans trois ruisseaux artificiels parallèles de laboratoire. De même, des tests de prédation ont été réalisés en aquariums.

\section{Les expériences dans le chenal de frai du Lapitxuri}

Ce ruisseau semi-naturel alimenté par le Lapitxuri, petit affluent de la Nivelle, dépend de la station d'hydrobiologie de l'INRA de St-Pée-sur-Nivelle. Avec $130 \mathrm{~m}$ de long et $2,8 \mathrm{~m}$ de large, il est divisé en 13 biefs identiques équipés de pièges à l'amont et à l'aval pour contrôler l'émigration (cf. BEALL et MARTY, 1983 a et $b$, pour plus de détails).

Le substrat est composé d'un mélange de graviers sur lequel sont disposées des pierres (15 à $25 \mathrm{~cm}$ de diamètre; $3 / \mathrm{m}^{2}$ ). La production naturelle d'invertébrés aquatiques dans le ruisseau est très abondante. Le débit est d'environ $50 \mathrm{l} / \mathrm{s}$ et chaque bief peut être grossièrement divisé en 3 zones de faciès différents : un profond amont, une aire médiane de radier et un profond aval. Le niveau, la température et les conditions météorologiques sont enregistrés en routine. 
Les poissons utilisés, alevins ou juvéniles des deux espèces, étaient issus d'oeufs de différentes origines provenant de géniteurs sauvages : Etat du Washington (USA) pour les saumons cohos; Origine Nivelle, Gave d'Oloron (France) ou Matamec (Québec, Canada) pour les saumons atlantiques (cf. BEALL et al., 1989, pour plus de détails). Pour les observations sur les alevins à l'émergence, les lots étaient introduits au stade oeufs ou alevins vésiculés à l'intérieur des graviers. Lors des confrontations entre alevins et juvéniles, ces derniers étaient installés dans les biefs avant l'émergence des premiers pour une bonne acclimatation. Des grilles permettant seulement le passage des alevins étaient disposées à l'entrée des pièges. Lors des contrôles de prédation, les juvéniles échantillonnés (5 à 20) pour examen des contenus stomacaux étaient remplacés par des individus équivalents stabulés à cet effet dans un bief particulier du chenal.

Sommaire des expériences dans le chenal du Lapitxuri (expériences 1, 2 et 3, cf. BEALL et al., 1989, pour plus de détails ; expériences 4 et 5 inédites).

- Expérience 1 : alevins à l'émergence des deux espèces.

Mise en charge de trois biefs avec 2000 oeufs oeillés de l'une ou l'autre espèce dans deux biefs et 1000 oeufs de chaque espèce dans le troisième. Inventaire de populations 2,5 mois plus tard dans chaque bief, divisé en trois parties par des filets lors de la pêche électrique : les profonds amont et aval et le radier intermédiaire.

- Expérience 2 : alevins de saumon atlantique à l'émergence et juvéniles de saumon coho âgés d'un mois après l'émergence.

Mise en charge de six biefs avec 2750 larves de saumon atlantique récemment écloses. Introduction le lendemain de 300 juvéniles de saumon coho âgés d'un mois ( $40 \mathrm{~mm}$ de longueur moyenne) dans trois de ces biefs. Analyse de la prédation sur deux échantillons de cohos prélevés pendant l'émergence des saumons atlantiques. Inventaire deux mois plus tard dans chaque type d'habitat.

- Expérience 3: alevins à l'émergence de saumon atlantique et juvéniles d'un an de saumon coho ou de saumon atlantique.

Introduction dans six biefs de 3000 oeufs oeillés de saumon atlantique. Dix jours plus tard, adjonction de 30 juvéniles d'un an de saumon atlantique dans deux biefs et de saumon coho dans deux autres biefs. Contrôle de la prédation en cours d'émergence des saumons atlantiques et lors de l'inventaire final, deux mois plus tard. atlantique.

- Expérience 4: alevins de saumon coho à l'émergence et juvéniles d'un an de saumon

Six biefs ont été ensemencés avec 3000 larves de saumon coho récemment écloses avec adjonction de 30 juvéniles d'un an de saumon atlantique 10 jours plus tard dans trois biefs. Contrôle de la prédation en cours d'émergence des saumons cohos et en fin d'expérience, 1,5 mois plus tard.

- Expérience 5 : juvéniles de trois mois après l'émergence de saumon atlantique et de saumon coho.

200 juvéniles de Salmo salar ont été introduits dans six biefs, dans lesquels ont été rajoutés respectivement $25,50,100,150,200$ et 300 juvéniles de cohos. Un septième bief sans coho constituait le témoin.

\section{Expériences en ruisseaux de laboratoire et aquariums}

Dans trois ruisseaux artificiels parallèles de laboratoire, des observations comportementales ont été réalisées sur des lots de poissons identiques à ceux utilisés pour les expériences 1 à 5 dans le chenal du Lapitxuri (cf. BEALL et al., 1989, pour plus de détails). La première expérience avec des alevins à l'émergence consistait à comparer 200 alevins de l'une et l'autre espèce dans deux ruisseaux avec 100 alevins de chaque espèce en mélange dans un 
troisième. La seconde expérience, basée sur le même principe, portait sur des alevins âgés de trois mois introduits à des densités plus réduites pour tenir compte de l'augmentation de taille des individus.

Pour analyser plus précisément le rôle de la prédation potentielle des juvéniles de Salmo salar sur les alevins de saumon coho, des tests ont été réalisés dans 6 aquariums de 600 । alimentés par de l'eau filtrée et équipés d'une pompe intérieure créant le courant $(6$ à $40 \mathrm{~cm} / \mathrm{s}$ ). La photophase $(10 \mathrm{~h})$ et la température $\left(12-14^{\circ} \mathrm{C}\right)$ restaient constantes. La nourriture naturelle était distribuée quotidiennement.

Au cours d'un premier test, 15 alevins émergeant de coho étaient déversés dans chaque aquarium et 3 juvéniles de $S$. salar étaient ajoutés 2 jours plus tard dans 4 aquariums. La position et l'activité des poissons étaient enregistrées quotidiennement pendant 15 jours et la survie contrôlée en fin d'expérience. Durant le second test, les alevins de coho étaient âgés de 15 jours.

\section{RÉSULTATS}

Seuls les principaux résultats susceptibles d'apporter les éléments de compréhension nécessaires à l'évaluation des conséquences d'une introduction accidentelle sont rapportés ici.

1 - Alevins des deux espèces au même stade de développement (expérience 1 et observations en ruisseaux artificiels de laboratoire)

Dans le chenal du Lapitxuri, la survie des juvéniles est bonne, environ $80 \%$, sans différences notables entre les biefs. La dévalaison est plus forte en situation d'allopatrie pour les deux espèces et elle reste plus prononcée chez le saumon atlantique. Les patterns de dévalaison sont similaires pour les deux espèces. La croissance ne semble pas affectée par la présence de l'autre espèce. Cependant, il existe une très forte différence de taille en faveur des saumons cohos, dès le départ.

Dans les ruisseaux artificiels de laboratoire dans lesquels les ressources spatiales et alimentaires sont nettement plus restreintes qu'au Lapitxuri, la présence des alevins de saumon coho a un effet défavorable sur les alevins de saumon atlantique : moindre observation d'alevins territoriaux, davantage de dévalaison, croissance significativement plus faible. L'examen détaillé des comportements des alevins montre que les cohos occupent majoritairement $(80$ à $85 \%$ ) des postes de chasse en pleine eau alors que les alevins de saumon atlantique sont beaucoup plus benthiques : davantage en position de nage stationnaire intermittente, près du substrat ou appuyés sur les pectorales au contact du substrat. Cette différence est très nette en situation de sympatrie comme d'allopatrie.

\section{2 - Alevins de saumon atlantique à l'émergence et juvéniles de saumon coho âgés d'un mois (expérience 2)}

Au cours de cette confrontation, les plus gros juvéniles de saumon coho ont une telle taille qu'ils sont en mesure d'ingérer les alevins émergeant de saumon atlantique. Ainsi, une survie beaucoup plus forte est observée dans les biefs témoins sans saumons cohos : $72,3 \%$ contre $45,5 \%\left(\chi^{2}=1217, P<0,005\right)$. Cependant, aucun des 90 estomacs de juvéniles de coho échantillonnés ne contient de traces de saumon atlantique. Ce dernier résultat infirme l'hypothèse de la prédation pour expliquer la moindre survie des alevins de saumon atlantique en présence de juvéniles de saumon coho.

Dans cette expérience, le pattern de dévalaison des alevins de saumon atlantique est influencé, notamment dans sa durée, par la présence des cohos puisque $95 \%$ de la dévalaison se produit en 32 jours dans les biefs où les espèces sont en compétition, contre 24 jours dans les biefs témoins (Fig. 1). De même, la dévalaison diurne est généralement réduite en présence des juvéniles de coho (Fig. 2). 

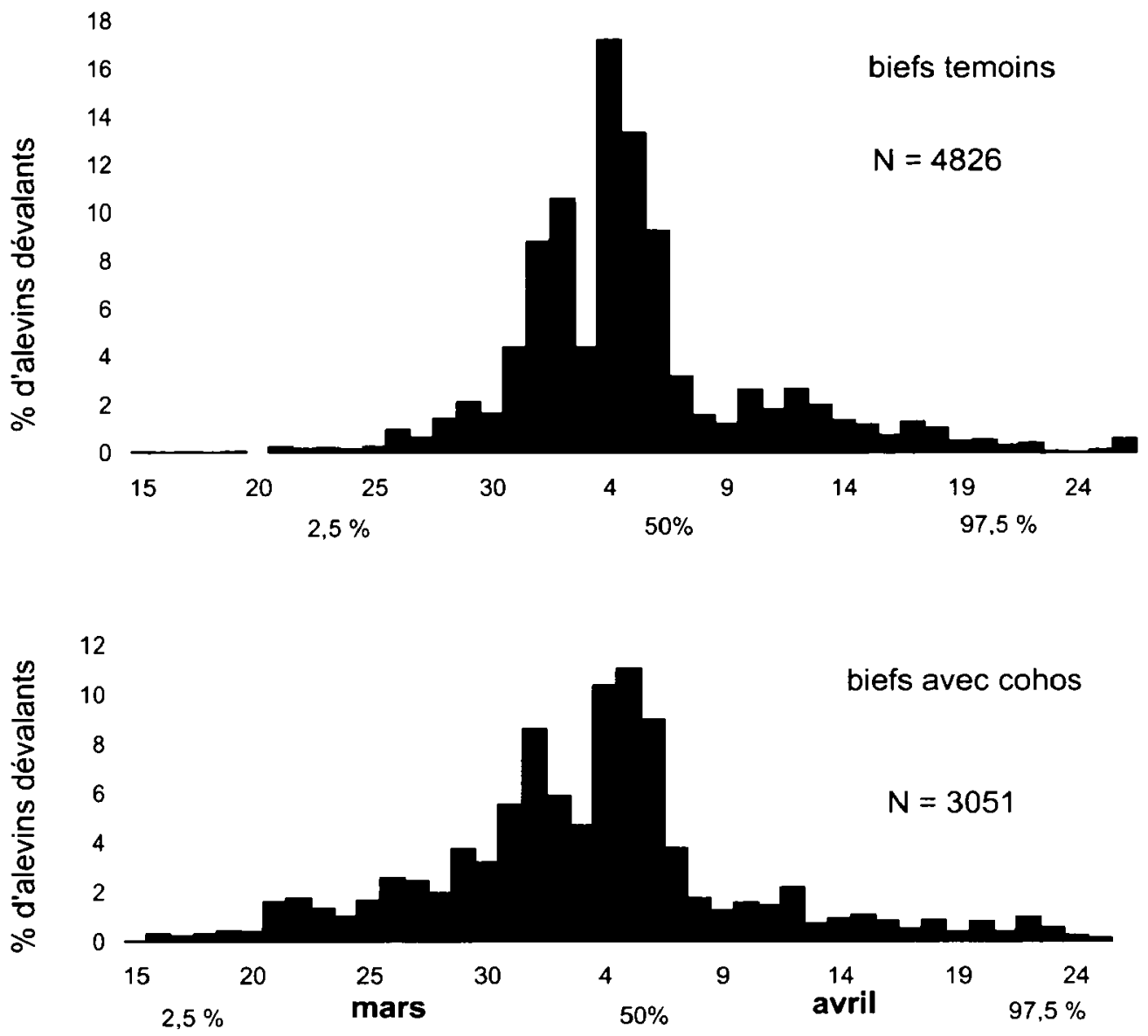

\section{Figure 1}

Patterns de dévalaison chez les alevins de saumon atlantique seuls (témoin) ou en présence de juvéniles de saumon coho dans le chenal de frai du Lapitxuri. Les histogrammes représentent l'évolution des pourcentages quotidiens de captures dans les pièges aval, par rapport à la totalité des captures enregistrées entre le 15 mars et le 26 avril. Les flèches indiquent les dates auxquelles $2,5,50$ et $97,5 \%$ des captures ont été effectuées.

Figure 1

Downstream moving patterns of Atlantic salmon fry alone (control) or in presence of coho salmon underyearlings in the Lapitxuri spawning channel. Histograms represent daily percentages of captures in downstream traps, in relation to total captures between 15 March and 26 April. Arrows show days when 2.5, 50 and $97.5 \%$ of total captures were made.

L'occupation des faciès " radier " ou "profond " en fin d'expérience est représentée sur la figure 3. La densité des alevins de saumon atlantique est bien corrélée avec la vitesse du courant et la profondeur : ils préfèrent les radiers (BEALL et al., 1989). Les juvéniles d'un mois de saumon coho se retrouvent très majoritairement dans les profonds où leur influence réduit significativement la croissance des alevins de saumon atlantique (Fig. 4).

3 - Alevins de saumon atlantique à l'émergence et juvéniles d'un an de saumon atlantique ou de saumon coho (expérience 3 )

Aucune prédation n'est observée de la part des juvéniles de saumon coho sur les alevins de saumon atlantique d'après les 60 contenus stomacaux analysés. Par contre, les tacons de saumon atlantique semblent exercer une prédation faible sur leurs conspécifiques à l'émergence : 2 alevins trouvés sur 56 estomacs examinés. 


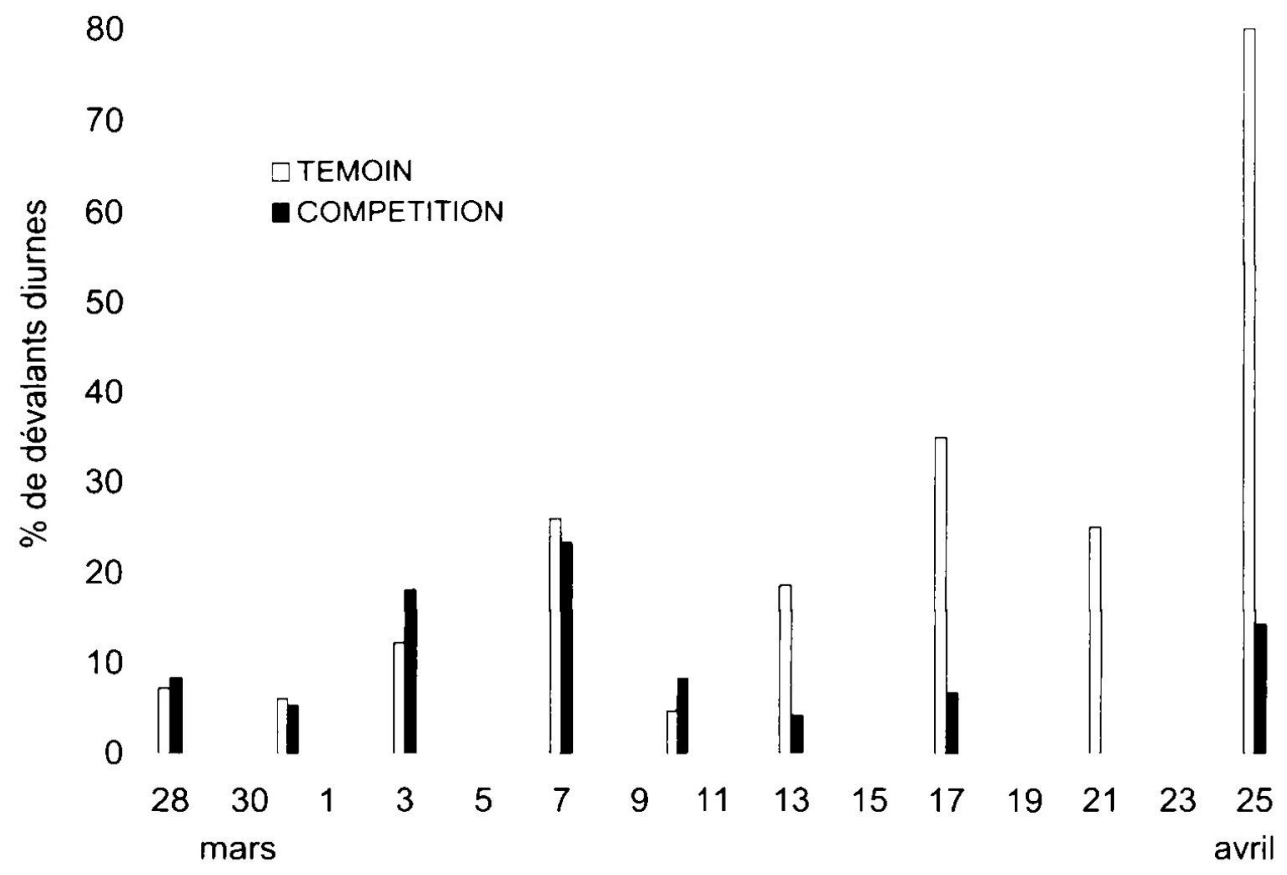

Figure 2

Proportions d'alevins dévalants de saumon atlantique durant le jour, en présence ou absence de juvéniles de saumon coho.

Figure 2

Proportions of Atlantic salmon fry moving downstream during the day, in presence or absence of coho salmon underyearlings.

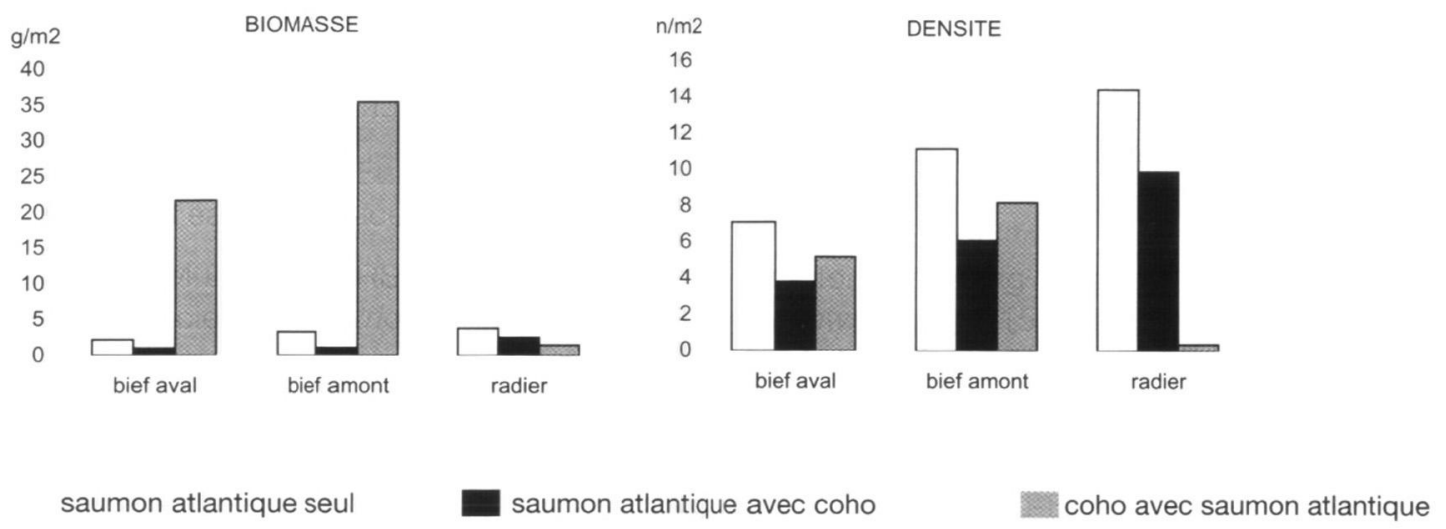

Figure 3

Densité et biomasse de juvéniles résidents de saumon atlantique ou de saumon coho seuls (uniquement pour le saumon atlantique dans les sections de contrôle) ou ensemble dans les différents faciès du chenal de frai du Lapitxuri : profond amont, profond aval et radier intermédiaire.

Figure 3

Density and biomass of resident Atlantic salmon and coho salmon underyearlings either alone (Atlantic salmon only in control sections) or together in different habitat types of the Lapitxuri spawning channel : upstream pool, downstream pool and middle riffle. 


$$
\begin{gathered}
\text { Poids (g) } \\
0,35 \\
0,3 \\
0,25 \\
0,2 \\
0,15 \\
0,1 \\
0,05 \\
0
\end{gathered}
$$

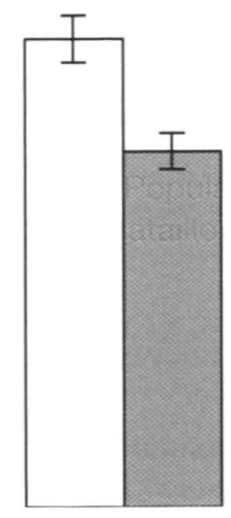

bief aval saumon atlantique seul

saumon atlantique avec coho

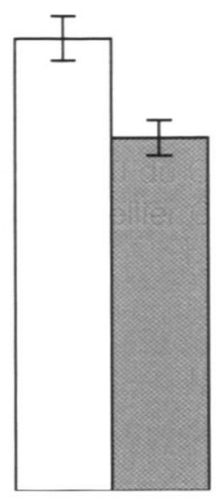

bief amont

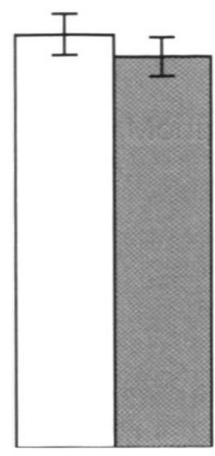

radier

Figure 4

Taille des alevins de saumon atlantique dans les faciès profond-amont, profond-aval et radier intermédiaire du chenal du Lapitxuri, en l'absence ou en présence de juvéniles de saumon coho.

\section{Figure 4}

Size of resident Atlantic salmon fry in upstream pool, downstream pool and middle riffle of the Lapitxuri channel, in the absence or presence of coho salmon underyearlings.

Les taux de piégeage diurnes et nocturnes d'alevins dévalants de saumon atlantique ont été considérés au cours de deux cycles quotidiens, juste avant et après le pic de dévalaison. Dans les biefs témoins, les alevins de saumon atlantique en situation d'allopatrie dévalent de jour à un taux moyen de $10,3 \%$. En présence de leurs congénères d'un an, ce taux est de $4,7 \%$. En présence des juvéniles de coho, la proportion moyenne tombe à 2,6\%. Tout se passe comme si la présence des juvéniles d'un an avait un effet inhibiteur sur l'activité diurne des alevins.

4 - Alevins de coho à l'émergence et juvéniles de saumon atlantique d'un an (expérience 4 et observations en aquariums)

Dans le chenal du Lapitxuri, une crue accidentelle a perturbé les résultats concernant la survie. Cependant, le taux de résidence, la densité, la biomasse et la croissance observés dans les biefs témoins en fin d'expérience sont plus élevés qu'en présence des juvéniles de saumon atlantique. Les échantillonnages de contenus stomacaux mettent en évidence la présence de 30 alevins de coho dans les 70 estomacs de saumon atlantique analysés.

Dans les aquariums à courant d'eau, la prédation exercée par les juvéniles d'un an de saumon atlantique sur les alevins de saumon coho est très forte à l'émergence : $100 \%$ des alevins capturés. Un mois plus tard, ces alevins semblent moins vulnérables : $50 \%$ sont capturés par les tacons de saumon atlantique.

5 - Juvéniles de saumon atlantique et de saumon coho (expérience 5 et observations en ruisseaux artificiels de laboratoire)

Au cours de cette expérience dans le chenal du Lapitxuri, la survie a été excellente dans tous les biefs (> $87 \%$ ). Chez le saumon atlantique, la dévalaison n'est pas significativement corrélée à la densité de juvéniles de saumon coho. Par contre, pour les juvéniles de saumon coho, un effet très fort de la densité de ces derniers s'exerce sur leur dévalaison, ainsi que sur leur croissance (Fig. 5 et 6 ). 


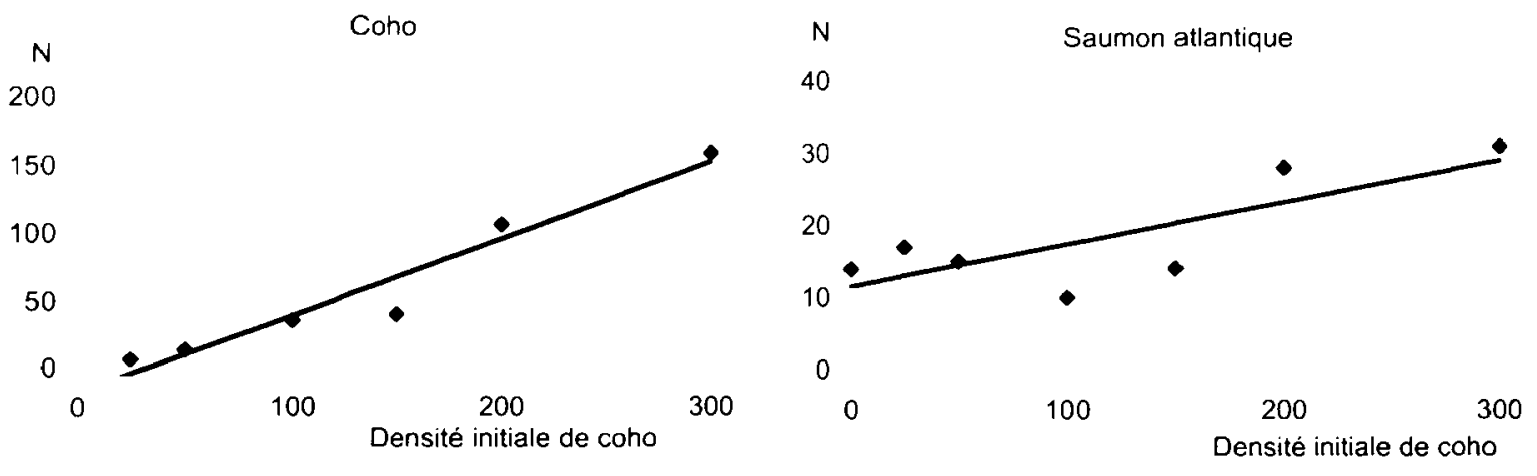

Figure 5

Nombre de juvéniles dévalants âgés de 3 mois de saumon atlantique et de saumon coho dans les sections du chenal du Lapitxuri, en fonction de la densité initiale de jeunes saumons cohos (pour le saumon atlantique : $y=11,57+0,05 x ; r=0,74$; pour le saumon coho : $y=-18,91+0,57 x$; $r=0,97)$.

Figure 5

Number of downstream moving three-months-old Atlantic salmon and coho salmon juveniles in the Lapitxuri channel sections, in relation to the initial density of three-months-old coho salmon juveniles (for Atlantic salmon : $y=11.57+0.05 x ; r=0.74 ;$ for coho salmon : $y=-18.91+0.57 x$; $r=0.97$.
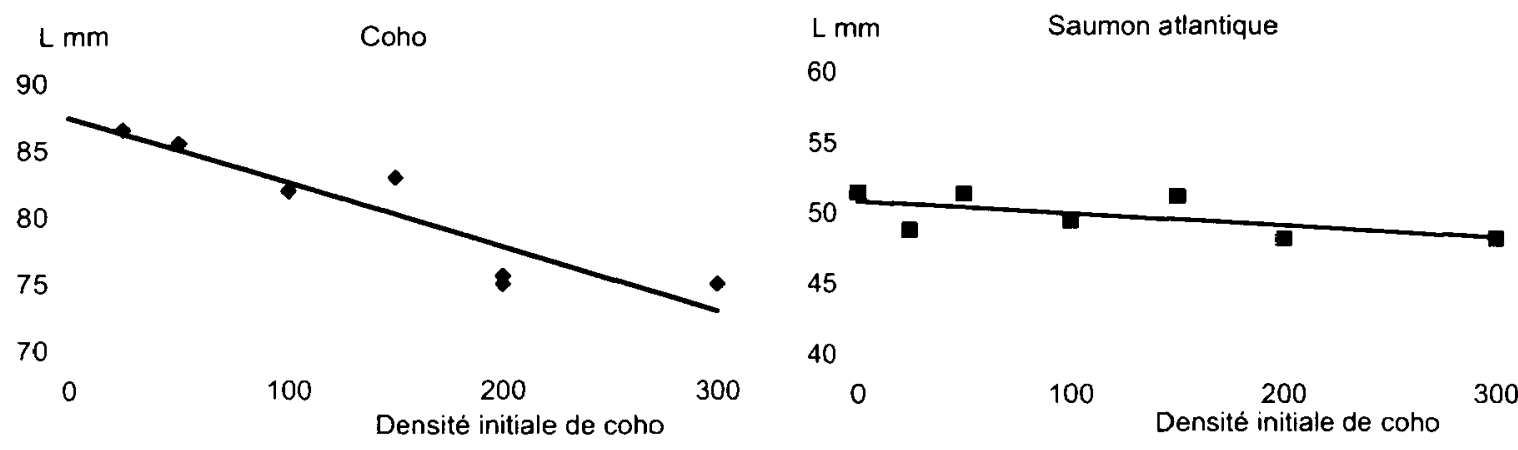

Figure 6

Longueur moyenne $(\mathrm{mm})$ des juvéniles de 3 mois résidents de saumon atlantique ou de saumon coho en fin d'expérience, en fonction de la densité initiale de saumons cohos juvéniles âgés de 3 mois.

Figure 6

Mean length $(\mathrm{mm})$ of resident Atlantic salmon or coho salmon juveniles at the end of the experiment, in relation to the initial density of three-months-old coho salmon juveniles.

A ce stade, les choix d'habitat ont évolué. Les juvéniles de saumon atlantique occupent alors tous les faciès disponibles avec la plus forte biomasse dans la zone profonde de l'amont des biefs. Il apparait clairement que les saumons atlantiques de cette taille $(50 \mathrm{~mm}$ de longueur moyenne) sont moins influencés par les juvéniles de saumon coho $(80 \mathrm{~mm}$ de longueur moyenne) que lorsqu'ils sont plus jeunes. 


\section{DISCUSSION}

Les compétitions interspécifiques entre différentes espèces de Salmonidés ont donné lieu à de nombreuses études, en particulier en Amérique du Nord où diverses espèces coexistent en sympatrie (NEWMAN, 1956; GIBSON, 1966; ANDRUSAK et NORTHCOTE, 1971 ; EVEREST et CHAPMAN, 1972 ; STEIN et al., 1972 ; GLOVA, 1984, 1987). En milieu naturel, les mécanismes de la compétition conduisent les individus de chaque espèce à tirer parti de leurs différences comportementales spécifiques, pour se réfugier dans la portion de l'environnement ou microhabitat dans laquelle ils sont les mieux adaptés : c'est le concept de ségrégation interactive appliqué aux poissons par NILSSON (1967). S'il ne coexiste pas dans la nature avec le saumon atlantique, le saumon coho peut s'observer en sympatrie avec la truite steelhead (Oncorhynchus mykiss) qui a des besoins écologiques et un cycle assez proche de ceux du saumon atlantique. Dans de telles situations, ALLEE $(1974,1982)$ a observé une ségrégation interactive en été, la truite steelhead occupant les radiers alors que les jeunes saumons cohos se retrouvaient dans les profonds. En ruisseau artificiel, GIBSON (1981) a observé expérimentalement le même type d'interactions, entre les juvéniles de saumon coho et de saumon atlantique.

Si l'on considère l'ensemble de nos expériences, il n'apparaît pas d'effet négatif évident de la présence des saumons cohos sur le développement des alevins de saumon atlantique. En fonction des situations, nos résultats sont difficiles à interpréter à cause des effets cumulés entre les influences de la compétition intraspécifique ou interspécifique et les différences interspécifiques.

Cependant, à partir de nos expériences, deux points importants ressortent :

- La taille des alevins de saumon coho est très supérieure à celle des alevins de saumon atlantique, au même stade de développement. Dès l'émergence, ils occupent en fonction de leur taille une portion d'habitat (ou strate par rapport au substrat) située au-dessus de celle utilisée par les alevins de saumon atlantique.

- Les alevins de saumon atlantique occupent les radiers, alors que ceux de saumon coho préfèrent les zones plus profondes. En présence des cohos, les jeunes saumons atlantiques sont cantonnés dans les radiers. Avec l'âge et la croissance en taille, ils préfèrent les radiers plus profonds ou l'entrée des profonds occupés par les juvéniles de coho (SYMONS et HELAND, 1978).

Cette situation est résumée schématiquement sur la figure 7 . Le ruisseau peut être représenté de l'amont vers l'aval en une succession classique de radiers suivis de profonds séparés par une zone de transition constituée par des radiers plus ou moins profonds et rapides. Les alevins de saumon atlantique à l'émergence occupent la zone de radiers peu profonds, qu'ils exploitent à partir de postes de chasse et de territoires pour la capture des invertébrés de la dérive. Les alevins de coho, beaucoup plus gros, utilisent préférentiellement les zones plus profondes, à courant modéré ou lent, où ils se positionnent en pleine eau, avec un comportement alimentaire plus pélagique comme SYMONS et MARTIN (1978) et GIBSON (1981) l'avaient observé. ALLEE (1982) avait aussi remarqué une stratification verticale du comportement des cohos par rapport aux truites steelhead plus près du fond.

Lorsqu'ils sont en compétition, les cohos influencent les alevins de saumon atlantique en les contraignant à quitter les zones de radiers profonds par dévalaison nocturne et restriction des activités durant le jour. Aucune prédation n'est enregistrée de la part des cohos. Dans les ruisseaux artificiels de laboratoire, le comportement cryptique des alevins de saumon atlantique en présence des cohos conduit à une diminution de la croissance et de la survie.

Lorsqu'ils sont plus âgés, les juvéniles de saumon atlantique sont moins sensibles à l'influence de l'autre espèce. Par ailleurs, les tacons d'un an de saumon atlantique exercent une prédation importante sur les alevins de coho, dont la vulnérabilité augmente avec leur comportement de nage plus pélagique. Les mèmes tacons d'un an exercent une prédation 


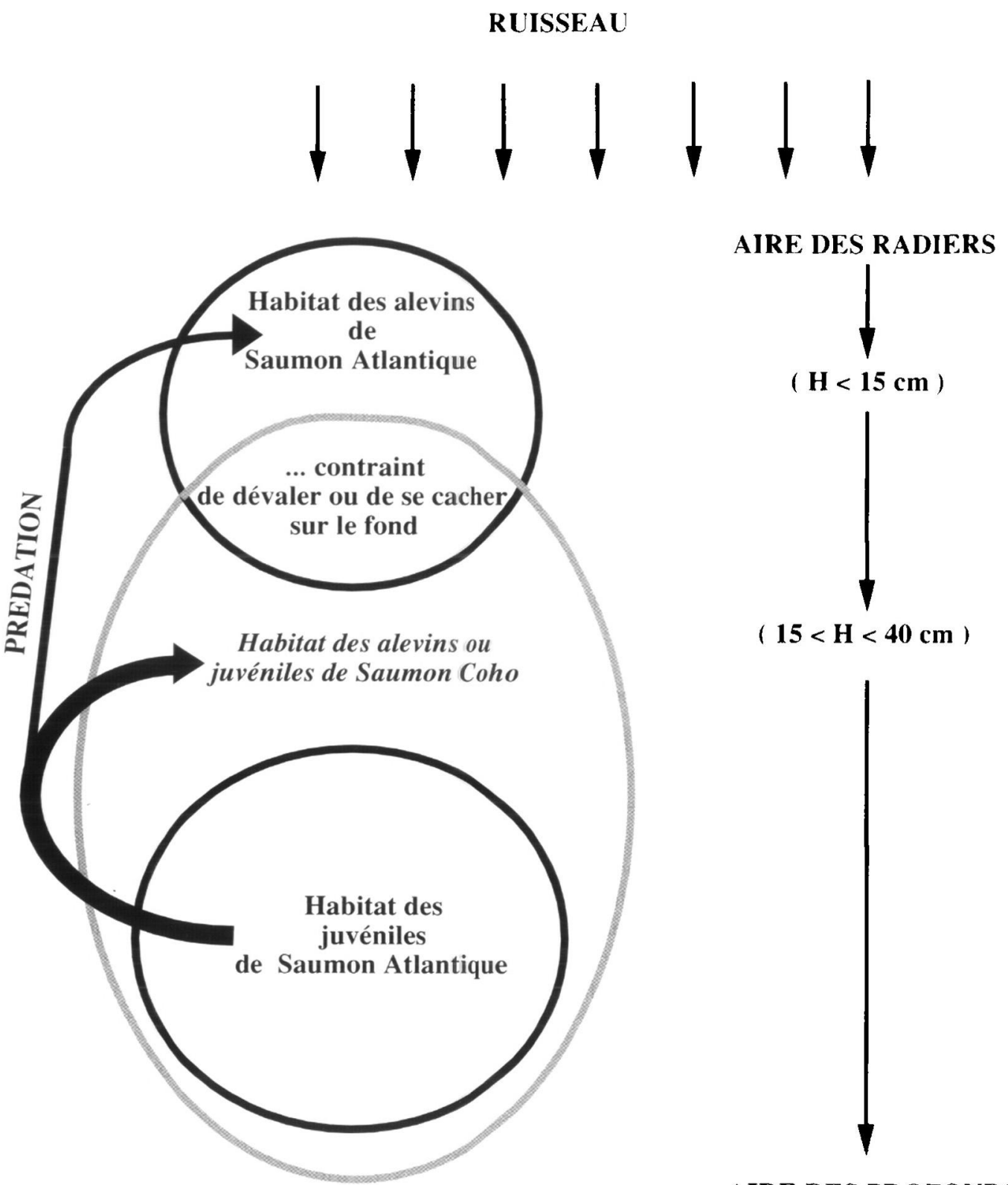

AIRE DES PROFONDS

\section{Saumon Atlantique}

\section{Saumon Coho}

Figure 7

Représentation schématique de la compétition pour l'habitat en ruisseau chez les alevins et juvéniles de saumon atlantique et de saumon coho.

Figure 7

Schematic representation of stream habitat competition in Atlantic salmon and coho salmon fry and juveniles.

faible sur leurs conspécifiques plus jeunes, sans doute parce qu'ils évoluent beaucoup plus près du fond. De plus, leur présence entraîne une augmentation de la dévalaison et une diminution de la croissance de leurs congénères alevins (expérience 3 ). 
L'expérience 5, destinée à tester l'influence d'une densité croissante de juvéniles de coho sur des juvéniles de saumon atlantique, démontre l'effet dominant de la compétition intraspécifique en accord avec les résultats d'ALLEE (1982) et GLOVA (1984). Le résultat s'explique par le fait que les juvéniles de la même espèce exploitent les mêmes ressources limitées d'un microhabitat différent de celui exploité par l'autre espèce en sympatrie, selon le principe de ségrégation interactive (HEARN, 1987).

\section{CONCLUSION}

En ruisseau naturel, sur les zones de frayères, même si les juvéniles de coho n'exercent pas de prédation ou n'éliminent pas directement les alevins de saumon atlantique, leur seule présence a un effet inhibiteur sur ces derniers. Cela se traduit par une croissance moindre et une restriction aux zones de radiers, par la mise en jeu des mécanismes de ségrégation interactive. Entre ces deux espèces, théoriquement jamais présentes ensemble dans la nature, une confrontation en milieu naturel peut avoir des résultats très variables selon les disponibilités du milieu, avec une coexistence plus ou moins avantageuse pour l'une ou l'autre espèce (HEARN, 1987). Dans tous les cas, la capacité d'accueil d'une rivière étant limitée, l'introduction d'une espèce affectera nécessairement le développement de l'espèce directement concurrente (ROSE, 1986).

Ces différentes études ont été conduites à partir de jeunes saumons cohos issus d'oeufs d'origine sauvage importés des États-Unis. Ces jeunes poissons se trouvaient donc en situation de colonisation d'un milieu très éloigné de leur aire d'origine. Une question demeure : est-ce que les sujets utilisés dans nos expériences étaient bien adaptés à ce nouveau milieu? Des sujets introduits depuis plusieurs générations auraient-ils eu le même comportement dans la même situation? On ne peut préjuger avec certitude de ce qui se passerait avec une population de saumon coho bien acclimatée dans une rivière française.

En définitive, nos résultats militent en faveur de l'abstention en matière d'introduction d'une espèce comme le saumon coho, même s'il est généralement admis que le risque de pullulation d'une telle espèce est faible comme chez la plupart des Salmonidés à migration lointaine.

\section{REMERCIEMENTS}

Cette recherche a reçu le soutien financier du CNEXO (contrats 81/2560/ $Y$ et $83 / 7248 / Y$ ). Nous remercions vivement C. MARTY ainsi que J.P. MARTINET et J.C. VIGNES pour leur assistance.

\section{BIBLIOGRAPHIE}

ALLEE B.J., 1974. Spatial requirements and behavioral interactions of juvenile coho salmon (Oncorhynchus kisutch) and steelhead trout (Salmo gairdneri). Unpubl. Ph. D. Thesis Ecology, Univ. Washington, Seattle, $160 \mathrm{p}$.

ALLEE B.J., 1982. The role of interspecific competition in the distribution of salmonids in streams in BRANNON E.L. and SALO E.O., Salmon and Trout migratory behavior Symposium, 111-122, School of Fisheries University of Washington, Seattle, Washington.

ANDRUSAK H., NORTHCOTE T.G., 1971. Segregation between adult cutthroat trout (Salmo clarki) and Dolly Varden (Salvelinus malma) in small coastal British Columbia lakes. J. Fish. Res. Board Can., 28, 1259-1268.

BEALL E., MARTY C., 1983 a. Reproduction du saumon atlantique, Salmo salar L., en milieu semi-naturel contrôlé. Bull. Fr. Piscic., 289, 77-83. 
BEALL E., MARTY C., 1983 b. Dévalaison et survie d'alevins de saumon atlantique, Salmo salar L., en milieu semi-naturel contrôlé. Bull. Fr. Piscic., 290, 135-148.

BEALL E., HELAND M., MARTY C., 1989. Interspecific relationships between emerging Atlantic salmon, Salmo salar, and coho salmon, Oncorhynchus kisutch, juveniles. J. Fish. Biol., 35 (suppl. A), 285-293.

EUZENAT G., FOURNEL F., 1981. L'introduction des saumons du Pacifique en France. Minist. Env. Cadre Vie, CSP, Dél. Rég. 1, Compiègne, 111 p.

EVEREST F.H., CHAPMAN D.W., 1972. Habitat selection and spatial interaction by juvenile chinook salmon and steelhead trout in two Idaho streams. J. Fish. Res. Board Can., 29, 91-100.

GIBSON R.J., 1966. Some factors influencing the distribution of brook trout and young Atlantic salmon. J. Fish. Res. Board Can., 23, 1977-1980.

GIBSON R.J., 1981. Behavioural interactions between coho salmon (Oncorhynchus kisutch), Atlantic salmon (Salmo salar), brook trout (Salvelinus fontinalis) and steelhead trout (Salmo gairdneri) at the juvenile fluviatile stages. Can. Tech. Rep. Fish. Aquat. Sci., 1029, $116 \mathrm{p}$.

GLOVA G.J., 1984. Management implications of the distribution and diet of sympatric populations of juvenile coho salmon and coastal cutthroat trout in small streams in British Columbia, Canada. Prog. Fish-Cult., 46, 269-277.

GLOVA G.J., 1987. Comparison of allopatric cutthroat trout stocks with those sympatric with coho salmon and sculpins in small streams. Envir. Biol. Fish., 20, 275-284.

HEARN W.E., 1987. Interspecific competition and habitat segregation among stream-dwelling trout and salmon : a review. Fisheries, 12, 24-31.

HELAND M., GAUDIN P., BARDONNET A., 1995. Mise en place des premiers comportements et utilisation de l'habitat après l'émergence chez les salmonidés d'eau courante. Bull. Fr. Pêche Piscic., 337/338/339, 191-197.

NEWMAN M.A., 1956. Social behavior and interspecific competition in two trout species. Physiol. Zool., 29, 64-81.

NILSSON N.A., 1967. Interactive segregation between fish species in GERKING S.D., The biological basis of freshwater fish production, 295-313, Blackwell Scientific Publications, Oxford and Edinburgh.

ROSE G.A., 1986. Growth decline in subyearling brook trout (Salvelinus fontinalis) after emergence of rainbow trout (Salmo gairdneri). Can. J. Fish. Aquat. Sci., 43, 1, 187-193.

SOLOMON D.J., 1979. Coho salmon in North-West Europe. Possible effects on native salmonids. Lab. Leafl. Fish. Lab. Lowestoft, 49, 21 p.

STEIN R.A., HALL J.D., REIMERS P.E., 1972. Social interaction between juvenile coho (Oncorhynchus kisutch) and fall chinook salmon (O. tshawytscha) in Sixes River, Oregon. J. Fish. Res. Board Can., 29, 1737-1748.

SYMONS P.E.K., HELAND M., 1978. Stream habitats and behavioral interactions of underyearling and yearling Atlantic salmon (Salmo salar). J. Fish. Res. Bd. Canada, 35, 175-183.

SYMONS P.E.K., MARTIN J.D., 1978. Discovery of juvenile Pacific salmon (coho) in a small coastal stream of New Brunswick, 487-489. 